\title{
APLICAÇÕES PARA A FERRAMENTA DE AVALIAÇÃO ONLINE QUIZ NA UNESC
}

\author{
Graziela Fatima Giacomazzo \\ Universidade do Extremo Sul Catarinense-gfg@unesc.net \\ Patricia Jantsch Fiuza \\ Universidade do Extremo Sul Catarinense - pjf@ unesc.net \\ Cleusa Ribeiro dos Santos \\ Universidade do Extremo Sul Catarinense - csa@ unesc.net \\ Almerinda Tereza Bianca Bez Batti Dias \\ Universidade do Extremo Sul Catarinense - bbd@ unesc.net \\ Evânio Ramos Nicoleit \\ Universidade do Extremo Sul Catarinense - ern@ unesc.net \\ Elisa Netto Zanette \\ Universidade do Extremo Sul Catarinense - enz@ unesc.net
}

\begin{abstract}
Resumo: Este artigo discute as aplicações da ferramenta de avaliação on-line Quiz incorporada ao Ambiente Virtual de Aprendizagem (AVA) de uma Instituição de Ensino Superior (IES). A ferramenta Quiz pode ser utilizada em diversos espaços acadêmicos e sua aplicação dependerá do objetivo proposto. Na Unesc, esta ferramenta tem sido utilizada em diferentes espaços e sua aplicabilidade vem acenando de forma promissora nos processos de avaliação na IES. Destaca-se o uso do Quiz na avaliação institucional; na avaliação da aprendizagem; na avaliação de projetos de extensão presenciais e a distância; em disciplinas da graduação presencial e disciplinas institucionais semipresenciais e a distância, projetos com $20 \%$ a distância por adesão (pilotagem), projetos de inovações tecnológicas como o uso da Lousa Digital Interativa (LDI) e no Programa de Monitoramento do Ensino - PROMA. A temática da avaliação e do uso dos recursos tecnológicos digitais no ensino superior é abordada neste relato onde se destacam a ferramenta Quiz, sua estrutura e funcionalidades, as aplicações no ensino superior e as considerações desta experiência.
\end{abstract}

Palavras-Chave: AVALIAÇÃO, QUIZ, ENSINO SUPERIOR, AMBIENTE VIRTUAL DE APRENDIZAGEM.

\section{APPLICATIONS QUIZ ONLINE EVALUATION TOOL AT UNESC}

Abstract: This paper discusses the applications of Quiz online evaluation tool incorporated into Virtual Learning Environment (AVA) of a Superior Education Institution (IES). The Quiz tool can be used in different academic areas and its implementation will depend on the proposed objective. At Unesc, this tool has been used in different spaces and its applicability has been doing promising in the evaluation process at the IES. We highlight the use of the Quiz on institutional evaluation; evaluation of learning; in the evaluation of presence and distance extension projects; in presential graduate disciplines and in semipresential and distance institutional disciplines; pilot projects with the distance modality by $20 \%$ adherence; projects of technological innovations as the use of Digital Interactive Whiteboards (LDI) and; Education Monitoring Program - PROMA. The tematichs of evaluation and use of digital technology resources in Superior Education is addressed in this report where we 
highlight the Quiz tool, its structure and features, applications in Superior Education and the considerations of this experience.

Keywords: EVALUATION, QUIZ, SUPERIOR EDUCATION, VIRTUAL LEARNING ENVIRONMENT.

\section{INTRODUÇÃO}

O tema da avaliação tem sido constantemente pesquisado e debatido nas universidades ao longo da história da educação superior. Refletir sobre avaliação na atualidade envolve analisar os instrumentos e os recursos disponíveis, entre eles, as tecnologias de informação e comunicação (TIC). É sobre este aspecto que o Grupo de Pesquisa Educação a Distância na Graduação (GPEAD) e o Setor de Educação a Distância (SEAD) da Universidade do Extremo Sul Catarinense (Unesc) vêm trabalhando, no contexto do ensino superior, em uma universidade comunitária, buscando potencializar inovações por meio do uso das tecnologias na educação.

Segundo Álvarez Méndez (2002, p. 16), “a avaliação faz parte de um continuum e, como tal, deve ser processual, contínua, integrada no currículo e, com ele, na aprendizagem". Segundo Leite (2010, p. 6) uma avaliação também se define como inovadora "[...] quando verificamos outras formas de interação, de relação, quer sejam entre sujeito e objeto, entre sujeito e TI". Com isto, introduz-se o uso de TIC nos processos avaliativos compreendendo que a inovação ocorre em consonância com as novas formas de interação. Apresenta-se neste artigo a aplicação de uma ferramenta on-line de avaliação denominada Quiz, as evidências com seu uso, bem como os fatores positivos destas aplicações:

A ferramenta Quiz pode ser utilizada em diversos espaços acadêmicos e sua aplicação dependerá do objetivo proposto. Na Unesc, esta ferramenta tem sido utilizada em diferentes espaços e sua aplicabilidade vem acenando de forma promissora nos processos de avaliação na IES. Pode-se citar o uso do Quiz na avaliação institucional; na avaliação da aprendizagem; na avaliação de projetos de extensão presenciais e a distância; em disciplinas da graduação presencial e disciplinas institucionais semipresenciais e a distância, projetos de $20 \%$ a distância, por adesão (pilotagem), projetos de inovações tecnológicas como o uso da Lousa Digital Interativa e no Programa de Monitoramento do Ensino - PROMA.

Descrevem-se então as possibilidades avaliativas com o uso do AVA e as suas respectivas ferramentas. Posteriormente discutem-se a ferramenta Quiz, sua estrutura e funcionalidades, as aplicações possíveis até o momento no ensino superior e as conclusões desta experiência.

\section{AMBIENTE VIRTUAL DE APRENDIZAGEM E AVALIAÇÃO}

A Unesc, por meio do Setor de Educação a Distância - SEAD e do Departamento de Tecnologia da Informação - DTI, disponibiliza desde 2003 a professores e acadêmicos o AVA. O AVA é um espaço acadêmico virtual que possibilita o encontro entre professor(es) e aluno(s) e suas produções. Destacam-se por meio da sua utilização as possibilidades de ampliação das interações entre os atores dos processos de ensino aprendizagem. O fluxo de informações aumenta consideravelmente e as diferentes formas de registro, produção e autoria podem ser incorporadas e socializadas neste ambiente.

Neste contexto, enfatizam-se as contribuições que o uso do AVA possibilita para o desenvolvimento da avaliação processual na Unesc nas modalidades presencial e a 
distância. A partir dos pressupostos elencados no texto "Avaliação Processual: a opção da Unesc ", organizado pela equipe do setor pedagógico, evidenciam-se os conceitos da avaliação processual institucional e como estes podem ser atendidos por meio do trabalho pedagógico desenvolvido no AVA e suas ferramentas:

- Avaliação como processo é contínua e necessita de acompanhamento do professor ao processo de construção do conhecimento pelo aluno. Todas as ações efetuadas no AVA são registradas, incluindo período e tempo; há ferramentas de administração da sala virtual para o professor com relatórios estatísticos da turma e individual por aluno.

- Avaliação e ensino vinculam-se no cotidiano do trabalho pedagógico e não apenas em momentos especiais. Esta perspectiva rompe com a visão linear de ensino e avaliação, adotando-se a concepção processual em que não se avalia somente ao aluno, mas todos os elementos que constituem a relação pedagógica. As dúvidas e reflexões surgem em diferentes momentos no processo de aprendizagem. E o AVA pode ser acessado a qualquer tempo e lugar, com isto pode-se potencializar a interação que ocorre além da sala de aula presencial. Há ferramentas de comunicação para: Chat, NetMeeting (reunião on-line) e Fórum, além da disponibilização de materiais para serem acessados de casa, do trabalho, ou outros espaços do cotidiano do acadêmico.

- É necessário o desenvolvimento de uma metodologia que reconheça e respeite as individualidades e priorize a apropriação de conhecimento. Constata-se que muitos alunos não se sentem à vontade para falar na sala de aula presencial: perguntar, tirar dúvidas. Estes usam melhor os fóruns virtuais. O AVA possibilita e oportuniza aos estudantes a participação escrita, de forma individualizada.

Ao inserir as tecnologias no contexto educativo, ampliam-se as possibilidades de interação e autonomia no processo de aprendizagem. No AVA o professor pode: organizar seus materiais em suporte digital - Webpage; manter agenda de compromissos acadêmicos e eventos atualizada; obter lista de contato dos alunos com foto; organizar grupos de trabalho para alunos; construir mural com notícias e assuntos do interesse do grupo; promover e realizar debates on-line em Fóruns Virtuais e NetMeeting; orientar e acompanhar trabalhos acadêmicos e outras ações de acordo com as necessidades e interesses da turma e, mais recentemente; realizar avaliação on-line (Quiz).

A publicação das produções da turma ao longo da disciplina no AVA constitui-se também no registro memorial do trabalho construído. Esta iniciativa potencializa a avaliação processual.

\section{FERRAMENTA QUIZ NA UNESC}

A equipe de desenvolvimento do AVA - SEAD e DTI, buscando responder às demandas por uma ferramenta de avaliação on-line para as disciplinas semipresenciais e presenciais com uso do AVA, optaram pela incorporação da ferramenta Quiz ao AVA em 2008/2. A ferramenta é integrada ao AVA da instituição e tem, portanto, seu acesso restrito aos usuários dos cursos e disciplinas da instituição.

A ferramenta Quiz, assim como o AVA, é um projeto Open Source, ou seja, de código aberto e distribuído sob licença GNU (GPL), usando a plataforma LAMP (Linux, Apache, MySql e PHP). Na programação foi utilizada linguagem de programação PHP

1 Texto disponível em: http://www.unesc.net/form_continuada/Texto_2.doc

V. $8 \mathrm{~N}^{\mathrm{o}} 3$, dezembro, 2010 
para carregamento das páginas dinâmicas e para armazenar suas informações de configuração para o banco de questões e respostas. O sistema gerenciador de banco de dados - SGBD - MySQL foi escolhido por sua robustez e rapidez em ambiente Web.

A integração da ferramenta Quiz com outros AVA, além do LearnLoop adotado na Unesc, tais como Moodle, TelEduc, Sakai, Tidia-Ae pode ser realizada, desde que estas sejam customizadas para cada plataforma.

Com o recurso do Ajax, gravam-se as respostas parciais das questões à medida que os alunos respondem ao Quiz, sem a necessidade de recarregar toda a página. Dessa forma, caso o usuário necessite sair e retornar ao Quiz por algum motivo ou problema durante a resolução da avaliação, as questões respondidas são automaticamente salvas.

No controle do tempo de solução da avaliação, caso o Quiz esteja configurado com um tempo limite para ser resolvido, optou-se pelo uso do Javascript. O HTML e o CSS entram respectivamente com as funções para estruturar e formatar as páginas web, possibilitando a exibição e leitura do conteúdo. ${ }^{2}$

Com relação às funcionalidades, o Quiz possibilita ainda que o professor ou usuário mantenha um banco de dados com questões categorizadas por disciplina e/ou temáticas conforme a Figura 1.

\begin{tabular}{|c|c|c|c|}
\hline \multicolumn{2}{|l|}{ Nome } & \multicolumn{2}{|c|}{ Ações } \\
\hline Behaviorismo & & Editar & Excluir \\
\hline Gestalt & & Editar & Excluir \\
\hline Psicanalise & & Editar & Excluir \\
\hline Psicologia Científica & & Editar & Excluir \\
\hline Psicossomática & & Editar & Excluir \\
\hline Transtornos Menais & & Editar & Excluir \\
\hline Nova Categoria & Reorganizar & & \\
\hline
\end{tabular}

Figura 1 - Tela Categorias Temáticas para Questões no Quiz.

O banco de questões criado fica disponível para todas as disciplinas e semestres para o professor podendo ser reutilizado e constantemente atualizado.

\begin{tabular}{|l|l|l|l|}
\hline Exibir Questóes: & Categorias: & Tipo: \\
\hline Todas & Todas & Todos & \\
\hline
\end{tabular}

\begin{tabular}{|c|c|c|c|c|c|}
\hline \# & Ord & Questão & Categoria & Tіро (Op.) & Ações \\
\hline 1 & & $\begin{array}{l}\text { Assinale a alternativa que classifica corretamente o } \\
\text { seguinte quadro patológico: }\end{array}$ & Transtornos Menais & Única (5) & (1) \\
\hline 2 & & $\begin{array}{l}\text { Assinale a alternativa que contém a afirmação } \\
\text { correta sobre a Psicanálise: }\end{array}$ & Psicanalise & Única (5) & 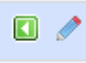 \\
\hline 3 & & $\begin{array}{l}\text { Assinale a alternativa que NÃO corresponde as } \\
\text { características corretas em relação à psicologia do } \\
\text { senso comum. }\end{array}$ & Psicologia Científica & Única (5) & 0 . \\
\hline 4 & & $\begin{array}{l}\text { Assinale a alternativa que possui o conceito que } \\
\text { designa uma compreensão imediata, enquanto uma } \\
\text { espécie de "entendimento interno", ou seja, um } \\
\text { processo equivalente ao do fechamento de uma }\end{array}$ & Gestalt & Única (5) & 10 . \\
\hline
\end{tabular}

2 Descrição técnica da ferramenta Quiz feita pelo desenvolvedor no DTI do AVA e ferramentas agregadas a este ambiente. Esp. Marcelo Mazon.

V. $8 \mathrm{~N}^{\mathrm{o}} 3$, dezembro, 2010 
Figura 2 - Tela Banco de Questões no Quiz do Docente.

Com isso é possível criar vários modelos de uma mesma avaliação, embaralhando a ordem das questões ou das respostas, além das opções de exibir retorno (número de acertos por aluno) e/ou solução (resposta correta) das questões. A ferramenta exibe telas bem desenhadas, amigáveis e de fácil entendimento, possuindo sistema de liberação de acesso aos alunos apenas quando o professor desejar.

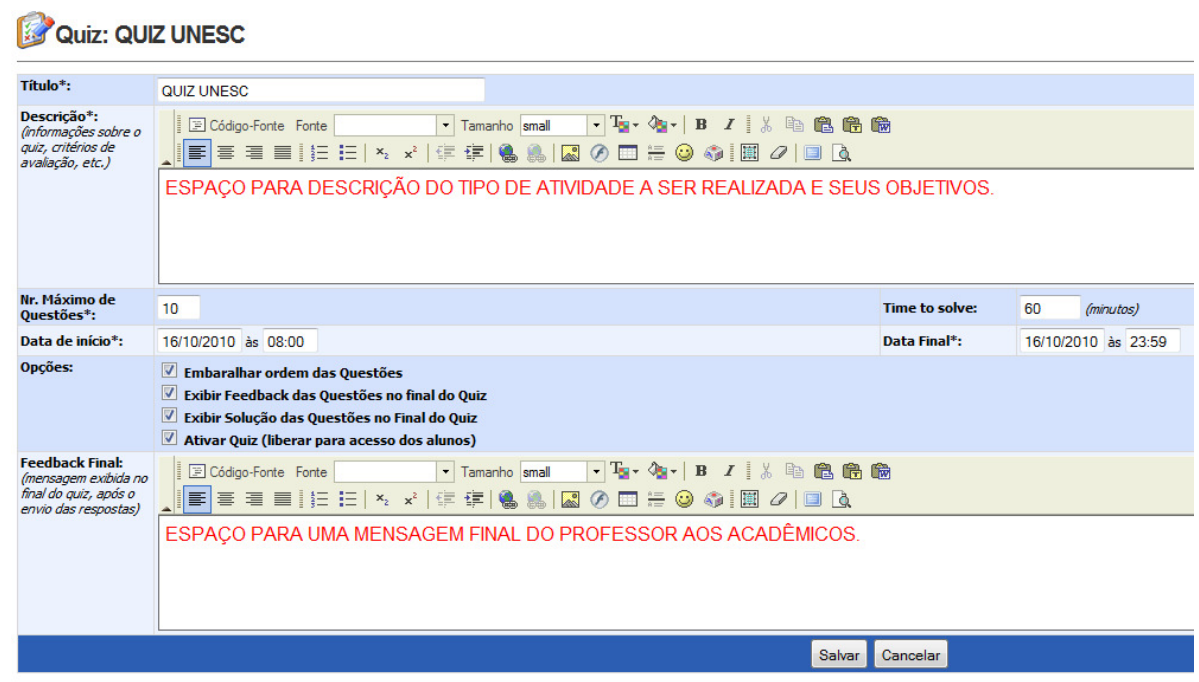

(*) Campos Obrigatórios!

Figura 3 - Tela de Configuração do Quiz pelo Docente.

Além do registro dos dados, questões e avaliações, o Quiz oferece ao professor diversos gráficos para analisar os rendimentos de cada turma ou aluno. Também é possível obter cálculo da média da turma e gráficos de avaliação. Por meio dos gráficos se pode também, visualizar a média do aluno $\times$ média da turma; o desenvolvimento do aluno por etapa; o desenvolvimento do aluno por avaliação; o desenvolvimento da turma; o desenvolvimento dos alunos em relação à média mínima exigida.

Pontuação Geral da Sala

\begin{tabular}{|c|c|c|c|c|c|c|}
\hline Nome & Codigo & $\begin{array}{l}\mathrm{Dt} / \mathrm{Hr} \\
\text { Inicio }\end{array}$ & $\begin{array}{c}\mathrm{Dt} / \mathrm{Hr} \\
\mathrm{Fim}\end{array}$ & Tempo & Qtd Questões & Score (\%) \\
\hline 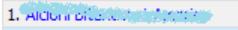 & 35 & 20/04/09 16:53:01 & 20/04/09 17:11:22 & $00: 18: 21$ & 4 & 100,00 \\
\hline 2. $n=-1$ & רידוב & 21/04/09 13:56:24 & $21 / 04 / 09 \quad 14: 08: 30$ & $00: 12: 06$ & 4 & 62,50 \\
\hline 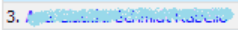 & $m$ & 22/04/09 08:29:01 & $22 / 04 / 0908: 41: 12$ & $00: 12: 11$ & 4 & 87,50 \\
\hline 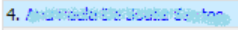 & joictiog & 22/04/09 08:02:10 & 22/04/09 08:10:09 & 00:07:59 & 4 & 37,50 \\
\hline 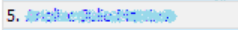 & west & 23/04/09 13:18:42 & 23/04/09 13:30:27 & $00: 11: 45$ & 4 & 50,00 \\
\hline 6. Farns: & ल. & $21 / 04 / 09$ 17:25:38 & 21/04/09 17:40:51 & $00: 15: 13$ & 4 & 100,00 \\
\hline 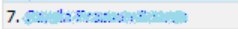 & : $: 2:$ & 21/04/09 11:27:11 & 21/04/09 11:43:31 & $00: 16: 19$ & 4 & 100,00 \\
\hline
\end{tabular}

Figura 4 - Exemplo de apresentação de desempenho por acadêmico. 
Pontuação Geral por Questão

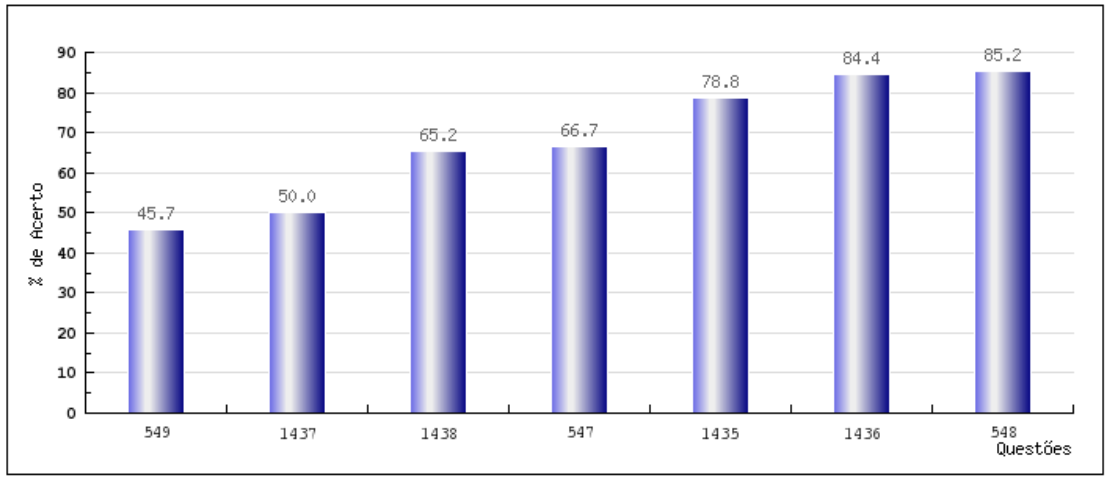

Figura 5 - Exemplo de apresentação de desempenho geral turma por questões.

Para o uso do Quiz pelos docentes, foram realizadas oficinas pela equipe do SEAD. As oficinas começaram a ser realizadas em 2009/1, sendo capacitados até 2010/01 64 docentes para uso da ferramenta Quiz. Contudo, muitos docentes, por familiaridade com as tecnologias exploram e usam as ferramentas sem a necessidade das oficinas.

Para compreender a dimensão do uso desta ferramenta apresentam-se os dados sobre o número de Quiz realizados: 344 em 2009/2; 482 em 2010/1; 117 até Setembro de 2010/02, chegando ao total de 943 instrumentos disponibilizados.

\section{APLICAÇÃO DA FERRAMENTA QUIZ}

A ferramenta Quiz até o momento tem sido utilizada nas seguintes aplicações: avaliação da aprendizagem; avaliação Institucional de disciplinas semipresenciais (20\%) e a distância (80\%); projeto PROMA; avaliação do projeto Lousa Digital Interativa e; pesquisas de opinião.

Descrevem-se a seguir as evidências com o uso desta ferramenta bem como os fatores positivos destas aplicações:

\subsection{Avaliação da aprendizagem}

Pode-se dizer que um dos aspectos que torna significativa a avaliação de aprendizagem com o uso do Quiz é os dados estatísticos gerados na apresentação dos resultados, tanto para docentes, quanto discentes.

A ferramenta apresenta os resultados tabulados por acadêmico, como também, o desempenho geral da turma por cada item questionado, inclusive em formato gráfico, conforme ilustrado nas Figuras 4 e 5.

Além disso, apresenta também o desempenho por questão da turma e individualmente. Essas possibilidades são relevantes no processo de aprendizagem, pois o acadêmico tem acesso ao seu desempenho individual podendo, dessa forma, fazer avaliação de seus acertos e dificuldades imediatamente. Ou seja: o estudante, ao terminar a avaliação, tem a oportunidade de revisar, obter a resposta ideal e procurar entendê-la.

Também é importante para o professor que pode, com base nos resultados gerais da avaliação, aferir em que aspectos são necessárias intervenções na(s) aula(s) seguinte(s), usando os resultados para os encaminhamentos necessários na avaliação processual. Ou ainda, se o Quiz for aplicado no início da aula como um diagnóstico, o docente poderá usar os resultados para debate no tempo subsequente, contribuindo significativamente no processo de aprendizagem. A Figura 6 ilustra uma das formas de visualização dos resultados por questão. 


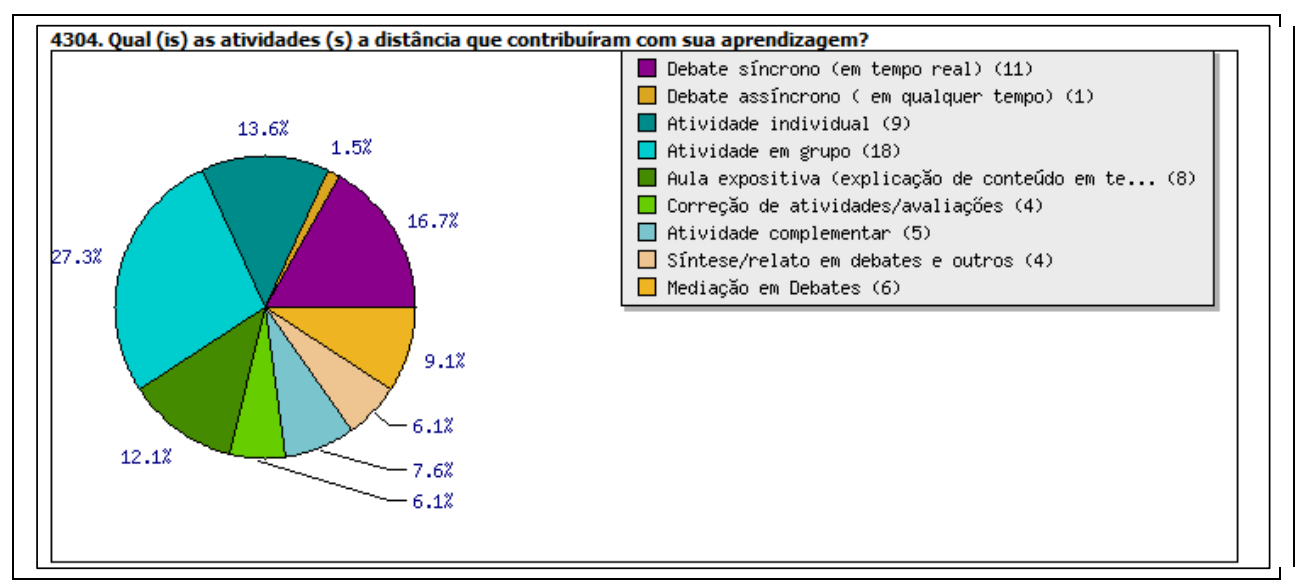

Figura 6 - Exemplo de apresentação de desempenho por questão.

A ferramenta vem sendo utilizada como forma de avaliação de aprendizagem em diversas disciplinas na Unesc. Nas disciplinas em que ocorrem $20 \%$ a distância, é utilizada de duas maneiras: avaliação parcial ao final de cada unidade de conteúdo ou aula e; avaliações integrais. A atividade avaliativa parcial tem sido bem aceita pelos discentes e docentes em função do retorno em tempo real. Dessa maneira, os próprios acadêmicos já retomam o conteúdo para solucionar as dúvidas em função das orientações - "dicas" (funcionalidade da ferramenta Quiz) - disponibilizadas pelo professor para auxiliar o estudante na resolução da atividade. Para os docentes, o retorno em tempo real, colabora no planejamento das estratégias pedagógicas posteriores. As avaliações são feitas nas aulas semipresenciais, a distância e presenciais nos laboratórios de informática da universidade.

De acordo com Marizete Santos et al (2009), o Quiz, pode ser entendido como uma espécie de jogo de perguntas e respostas que pode ser amplamente utilizado como ferramenta de retorno aos alunos na modalidade a distância. Pelo seu caráter interativo, pode estimular diferentes estilos de aprendizagem, contribuindo para o processo autoavaliativo dos educandos tanto na presencialidade como na modalidade de Educação a Distância (EaD).

Nas atividades avaliativas integrais, aqui consideradas as avaliações com peso 10 , o Quiz tem sido utilizado com eficácia tanto em atividades a distância, em que o acadêmico tem determinado tempo de realização da avaliação, mas tem flexibilidade quanto ao lugar, como também em atividades presenciais, usando os laboratórios de informática na instituição.

$\mathrm{O}$ resultado tem sido promissor em função da praticidade já discorrida anteriormente sobre a tabulação e a apresentação dos resultados, bem como também pela ferramenta possibilitar a contextualização das questões, o uso de imagens, textos, planilhas e outros recursos. Além disso, possibilita respostas em dois formatos: opção única ou múltipla escolha - o que permite um conjunto razoável de combinações a fim de não tornar a avaliação monótona, repetitiva ou com baixo nível de complexidade. 


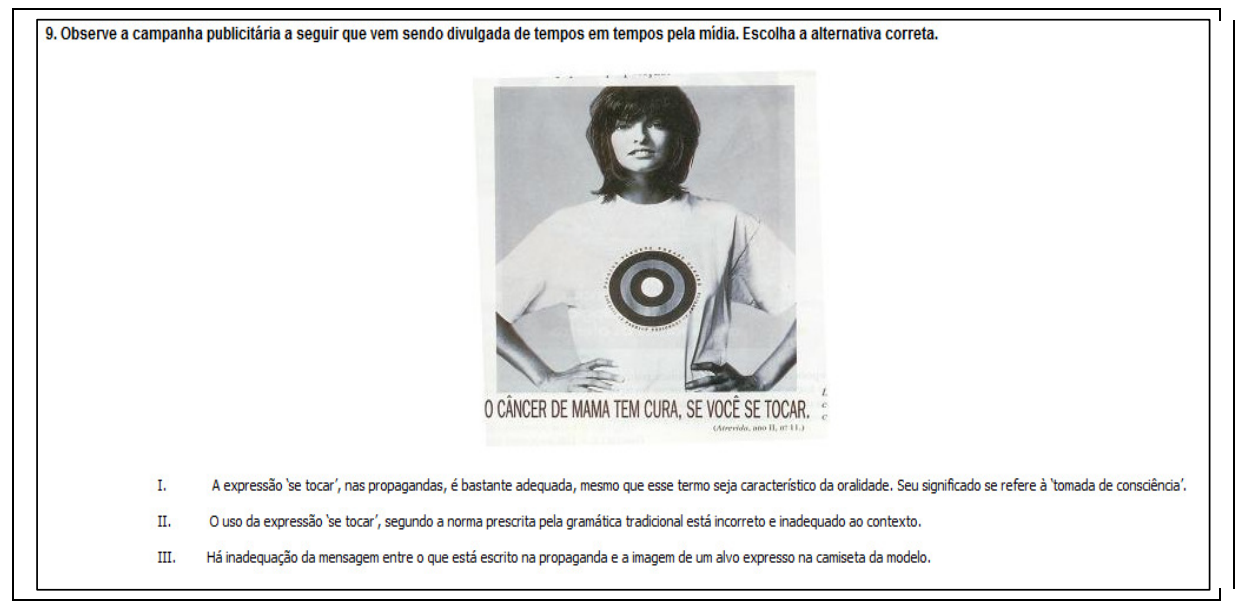

Figura 7 - Exemplo de pergunta usando imagem.

\subsection{Avaliação Institucional de Disciplinas Semipresenciais e a Distância}

A ferramenta Quiz, em função de estar inserida no AVA, vem sendo utilizada na pesquisa de avaliação das disciplinas em que institucionalmente ocorrem $20 \%$ ou mais de sua carga horária a distância. Na Unesc, ocorre nas disciplinas de Produção e Interpretação de Textos (PIT) e Metodologia Científica e da Pesquisa (MCP).

Há três semestres que se utiliza a ferramenta para esta avaliação conforme ilustra o quadro a seguir, nas disciplinas de PIT e MCP. Do semestre 2009/1 para 2010/1, houve um crescimento geral em torno de $40 \%$. Tal crescimento tem um limite em função do número de cursos que tem as duas disciplinas em sua grade curricular, que pode mudar a cada semestre. Contudo, esse crescimento é interessante, já que o SEAD, em conjunto com o Setor de Avaliação Institucional (SEAI), oferece ao professor duas possibilidades: instrumento impresso ou no Quiz. Logo, percebe-se a preferência dos docentes e discentes pela pesquisa utilizando a ferramenta on-line.

\begin{tabular}{|c|c|c|c|}
\hline Semestre/Disciplina & $2009 / 1$ & $2009 / 2$ & $2010 / 1$ \\
\hline PIT & 13 & 16 & 22 \\
\hline MCP & 30 & 26 & 30 \\
\hline
\end{tabular}

Quadro 1 - Número de cursos que usaram o Quiz na avaliação da disciplina.

Em relação às questões do instrumento, avaliou-se desde a modalidade de ensino, as ferramentas utilizadas no AVA, acesso, passando pelo grau de comunicação e interação entre os professores e acadêmicos, até o grau de satisfação do estudante com as ferramentas, atividades e aprendizagem.

\subsection{Programa de Monitoramento do Ensino - PROMA}

O PROMA contempla várias ações/atividades buscando a melhoria da aprendizagem do aluno. Foi idealizada com foco na avaliação processual, concepção esta adotada pela Unesc e compreendida como um movimento contínuo e que inclui toda a comunidade acadêmica envolvida no processo de ensino e aprendizagem. O PROMA foi estruturado por um grupo de professores da área das engenharias, a partir das fragilidades detectadas na aprendizagem dos acadêmicos nas disciplinas eixos nos cursos da Unidade Acadêmica, Ciências, Engenharias e Tecnologias (UNACET) da Unesc. 
Por meio de estudos realizados, observações efetuadas e resultados de avaliações dos alunos nas disciplinas, em específico, de Cálculo Diferencial e Integral e Álgebra, detectou-se a importância de se desenvolver ações mais eficazes e que contribuíssem para sanar e/ou minimizar as dificuldades de aprendizagem. Assim, privilegiou-se o Quiz como uma ferramenta de avaliação que possibilitasse um retorno imediato a professores e alunos das situações de aprendizagem. Assim, o Quiz passou a funcionar como ferramenta de retorno ao aluno, estreitamente ligada aos fluxos de avaliação processual tão importante no processo de aprendizagem.

Nesta perspectiva, foi solicitado ao SEAD, formação para o uso da ferramenta Quiz. Esta ferramenta contribuiria com a proposta de exercícios e problemas a serem resolvidos, pelo aluno, no AVA, com posterior correção e estatística de acertos. A ferramenta Quiz possibilitou que as provas do PROMA passassem a ser realizadas nos computadores em uma sala de informática, sem dispêndio algum de papel e agilidade de tempo de correção para os professores. Além disso, esta ação mobilizou outros docentes a utilizarem a ferramenta e os procedimentos do PROMA em suas disciplinas.

É interessante destacar que a interação SEAD/PROMA se mostrou produtiva agregando valor e conhecimento para ambos, disseminando esta experiência para outros espaços na instituição.

Conforme o professor Sérgio Souto Rocha (2008), coordenador do PROMA:

\begin{abstract}
Sabemos que os frutos efetivos desse trabalho só serão colhidos em seu verdadeiro teor a médio e longo prazo, mas a oportunidade que nos é dada de aprender e construir a linguagem de ensino mais atual e adequada aos padrões atuais é ímpar e nossos esforços são intensos e contínuos para estarmos à altura da oportunidade que nos é apresentada.
\end{abstract}

Um dos destaques do programa é o banco de questões e de provas, que possibilita manter as questões arquivadas e por tema, facilitando o trabalho de elaboração de provas e outras avaliações. Atualmente o PROMA possui um banco com mais de mil questões, sendo desenvolvido nos cursos de Engenharia de Materiais; Engenharia Civil; Engenharia Ambiental; Engenharia Química e Arquitetura e Urbanismo da Unesc.

\title{
4.4. Projeto Lousa Digital Interativa (LDI) e Pesquisa de Opinião
}

$\mathrm{O}$ processo de discussão e inclusão das $\mathrm{LDI}^{3}$ nas práticas pedagógicas situa-se como inovações tecnológicas nos cursos de graduação da Unesc. O projeto integra as políticas institucionais de fomento à cultura em $\mathrm{EaD}$ e formação do acadêmico para e com o uso de TIC. Para a avaliação deste projeto utilizou-se a ferramenta Quiz. A funcionalidade da ferramenta e a forma de apresentação dos dados facilitaram e dinamizaram o andamento do projeto, posto que os resultados e sugestões geraram modificações implementadas em tempo real. A ferramenta Quiz foi utilizada como instrumento de pesquisa integrado ao recurso de fórum no AVA para comentários e sugestões dos acadêmicos e docentes. Participaram desta pesquisa com uso do Quiz inicialmente, sete (07) professores e trezentos (300) acadêmicos de sete (07) disciplinas de três (03) cursos de graduação da UNACET (Engenharia Química, Engenharia Ambiental e Engenharia de Materiais). Atualmente, participam docentes e discentes representantes das (04)

\footnotetext{
3 Para mais informações este projeto está relatado no artigo: Construindo Novas Interações: AVA e Lousa Digital Interativa no Ensino Superior publicado na revista RENOTE: v. 8 n. 2, julho, 2010.
} 
Unidades Acadêmicas da Instituição. Com relação à pesquisa de opinião, constata-se que o Quiz também se mostra funcional, colaborando com a coleta de dados de forma ágil e eficiente pela sua rapidez na organização dos instrumentos, análise e divulgação dos dados da avaliação.

\section{CONCLUSÕES}

As possibilidades avaliativas com o uso do Quiz agregado ao AVA e suas respectivas ferramentas, ao serem utilizadas, a partir da concepção de avaliação institucional, aqui definida com avaliação processual, contribuem significativamente para melhorar de forma integrada às demais formas de avaliação presencial, semipresencial e a distância.

Neste contexto se insere a ferramenta Quiz na Unesc, assumindo um significado pedagógico, como possibilidade de tornar mais efetivos os resultados avaliativos, e qualificando o processo ensino aprendizagem. Como exemplo desta experiência, o monitoramento e acompanhamento da aprendizagem se configuraram como um dos desafios pedagógicos propostos pela coordenação de ensino da UNACET descritos no projeto PROMA.

Com relação à estrutura e funcionalidades, a ferramenta Quiz, está disponível para qualquer docente da instituição e vem sendo melhorada continuamente pela equipe do SEAD em parceria com o DTI e GPEAD.

Além da ferramenta Quiz, que destacou-se por sua estrutura, funcionalidades e aplicações, há outras ferramentas de geração e/ou disponibilização de questionários tais como Hot Potatoes e Lime Survey. Entretanto, neste estudo, não foram realizadas análises comparativas entre estas e o Quiz, pois o foco do trabalho era a avaliação online incorporada ao Ambiente Virtual de Aprendizagem (AVA). Contudo, poderão ser realizadas futuramente análises comparativas com mais propriedade a partir dos resultados encontrados com a aplicação do Quiz agregado ao AVA da UNESC.

Atualmente, a equipe de desenvolvimento, empenha-se na organização do banco de questões, que possibilitará o armazenamento, recuperação, seleção e uso por nível de complexidade, além de estar pesquisando novos recursos para a elaboração de questões abertas (qualitativas).

Foram apresentadas as aplicações disponíveis até o momento no ensino superior e as conclusões desta experiência. Contudo entende-se que outras aplicações, de acordo com os interesses e as necessidades de cada instituição, projeto e disciplina podem ser implementadas. A reflexão sobre as diversas experiências aqui relatadas prospecta a ampliação do uso das ferramentas do AVA, em especial da ferramenta Quiz, nas disciplinas e também nos projetos desenvolvidos nas UNA, setores e cursos da IES.

\section{REFERÊNCIAS}

MÉNDEZ, Juan Manuel Álvarez. Avaliar para conhecer, examinar para excluir. Porto Alegre: Artmed, 2002.

LEITE, Denise (Org).Inovação, Avaliação e Tecnologias de Informação. Porto Alegre: Pacartes, 2010.

ROCHA, Sergio Souto. Relatório de Atividades do Programa de Monitoramento do Ensino - PROMA. Universidade do Extremo Sul Catarinense- Unesc. Unidade Acadêmica de Ciências, Engenharias e Tecnologias -UNACET. Criciúma: dez. 2008. 
SANTOS Marizete et al. Quiz como Ferramenta de Feedback e Autoavaliação em Educação a Distância. Departamento de Estatística e Informática (DEINFO) Coordenação de Educação a Distância / Universidade Federal Rural de Pernambuco (UFRPE). Recife - PE: 2009. 